\title{
Slow-Release Technique of Dexamethasone Implant
}

\section{Mayur Kulkarni*, Divyansh Mishra and Mahesh Shanmugam}

Vitreo-Retina and Ocular Oncology Department, Sankara Eye Hospital, Bangalore, India

*Corresponding Author: Mayur Kulkarni, Vitreo-Retina and Ocular Oncology Department, Sankara Eye Hospital, Bangalore, India.
Received: March 26, 2021

Published: April 21, 2021

(C) All rights are reserved by Mayur Kulkarni., et al.

\begin{abstract}
Dexamethasone implant $\left(\mathrm{Ozurdex}^{\circledR}\right)$ is a sustained-release Intravitreal implant which contains dexamethasone $0.7 \mathrm{mg}$ in a drug delivery system called 'NOVADUR'. Certain cases may require dexamethasone implant injection along with vitrectomy, however, there is a lot of confusion, misconception and fear regarding the use of ozurdex implant in vitrectomized eyes [1]. In a vitrectomized eye where vitreous gel is absent, the chance of iatrogenic retinal injury is always there if adequate precautions are not taken while injecting the implant. Aim of the article is to demonstrate an in vivo safe technique of slow injection of the dexamethasone implant into vitrectomized eye along with vitrectomy surgery or in post-vitrectomy eye where only ozurdex implant is planned.
\end{abstract}

Keywords: Dexamethasone Implant; Ozurdex; NOVADUR

\section{Introduction}

Combined pars plana vitrectomy and intravitreal dexamethasone implant $\left(\right.$ Ozurdex $\left.^{\circledR}\right)$ may be effective in reducing macular edema in patients with various underlying ocular diseases like central retinal vein occlusion, diabetic macular edema, hemi-retinal vein occlusion, proliferative vitreoretinopathy and retinal detachment [2].

In the vitrectomized eye, usually vitreous gel is replaced by balanced salt solution (BSS) during surgery and aqueous subsequently which changes the intraocular biological conditions as well as the physical properties [3]. Although there are some studies showing similarity in pharmacokinetics of Ozurdex between vitrectomized and non-vitrectomized eyes [4] with clinical improvement of macular edema in various diseases $[5,6]$ the kinematics of ozurdex implant in vitrectomized eyes, have not been reported. Inappropriate technique of Ozurdex injection in the vitrectomized eyes can lead to complications like accidental retinal injury and vitreous hemorrhage [7].

Here with we describe our modification of slow release of dexamethasone implant in a case of vitrectomy combined with dexamethasone implant injection.

\section{Methodology \\ Surgical technique}

Once a 3 port pars plane $23 \mathrm{G}$ vitrectomy is completed, one of the superior vitrectomy port is removed as per the surgeons dominant hand and the dexamethasone implant injector is inserted through the active sclerotomy. The applicator is inserted intra ocularly in a controlled manner with bevel up position, until almost it is completely buried up to the silicone sleeve. The tip of the injector should face towards the posterior pole. Now keep the thumb on posterior one third part of the actuator button (Figure 1) and 
press it gradually and gently so that the implant is slowly injected avoiding sudden propulsion (Figure 2). Slow and gradual continuous pressure needs to be applied so the implant ejection process is slow and smooth. The aim should be to keep the gentle pressure on the actuator as slow and gentle as possible and not to have a sudden jerk/click. Confirm the complete expulsion of the implant with visible ejection of the implant pellet into the vitreous cavity during vitrectomy surgery (Video link) or with the click sound in postvitrectomized eyes where only ozurdex implant is planned.

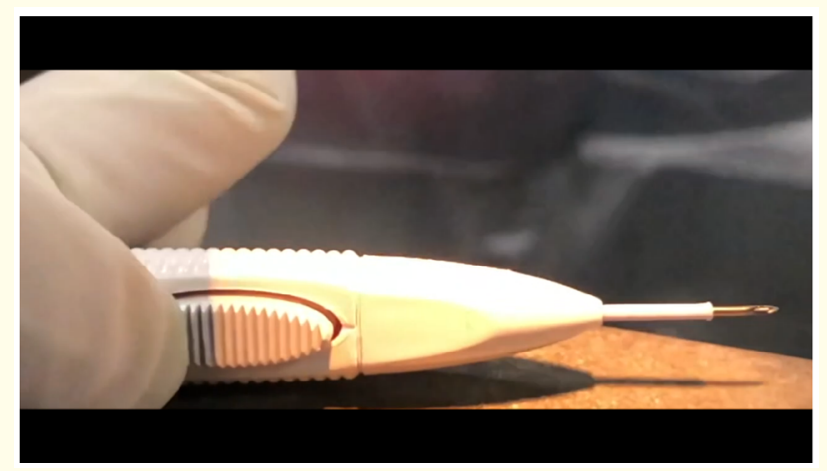

Figure 1: Slow and gradual continuous pressure needs to be applied over the back part of actuator, so the implant ejection process is slow and smooth.
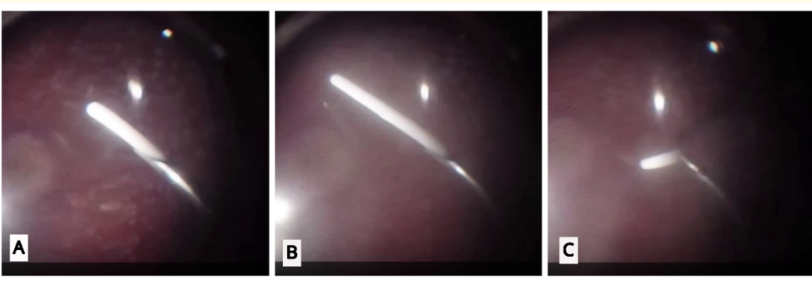

Figure 2: Demonstration of slow release of Ozurdex implant in vitrectomized eye avoiding sudden propulsion.

\section{Results and Discussion}

In cases of vitrectomized eyes as the eye is aqueous filled and there will not be any resistance once the implant pellet is suddenly injected and thus increasing the possibility of causing iatrogenic injury to various intraocular structures. A study by Krambeer., et al. studied the relation between the speed of applicator button depression and the speed of pellet injection into a calibrated ex vivo water bath [1]. They concluded that the force of the pellet insertion and mean impact velocity is significantly higher in the fast injection group as compared to the slow injection group [1].

A study by Ronakorn Panjaphongse., et al. analysed the kinematics of an Ozurdex implant, after its injection in a balanced salt solution (BSS) ex vivo at different release angles to simulate its movement in BSS/aqueous-filled eyes [8]. They concluded that an implant injected at a flatter angle tends to travel farther in the horizontal plane and has more initial velocity [8].

Our technique describes in vivo demonstration of the slow release of the dexamethasone implant with gradual continues pressure on the posterior one third part of the actuator button.

\section{Conclusion}

Ozurdex pellet implant injection by gradual and continues pressure over the back of the actuator button can potentially reduce the risk of retinal injury in vitrectomized eyes.

Video Link: https://drive.google.com/file/d/1kZEyDGPHXZxfB wCUo9cdCROmce7R695z/view?usp=sharing.

\section{Bibliography}

1. Krambeer CJ., et al. "A New Suggested Strategy for Safe Injection of Ozurdex". Ophthalmic Surgery, Lasers and Imaging Retina 50.2 (2019): e23-e25.

2. Zheng A., et al. "Combined vitrectomy and intravitreal dexamethasone (ozurdex) sustained-release implant". Retina 36.11 (2016): 2087-2092.

3. Lee SS., et al. "Vitreous VEGF clearance is increased after vitrectomy". Investigative Ophthalmology and Visual Science 51 (2010): 2135-2138.

4. Chang-Lin JE., et al. "Pharmacokinetics of a sustained-release dexamethasone intravitreal implant in vitrectomized and nonvitrectomized eyes". Investigative Ophthalmology and Visual Science 52 (2011): 4605-4609.

5. Ada'NA., et al. "Dexamethasone intravitreal implant for treatment of uveitic persistent cystoid macular edema in vitrectomized patients". Retina 33 (2013): 1435-1440. 
6. Boyer DS., et al. "Dexamethasone intravitreal implant for treatment of diabetic macular edema in vitrectomized patients". Retina 31 (2011): 915-923.

7. Lee SM., et al. "Retinal injury following intravitreal injection of a dexamethasone implant in a vitrectomized eye". International Journal of Ophthalmology-IJO 10.6 (2017): 1019-1020.

8. Panjaphongse R., et al. "Kinematic study of ozurdex injection in balanced salt solution: modeling the behavior of an injectable drug delivery device in vitrectomized eyes". Journal of Ocular Pharmacology and Therapeutics 31.3 (2015): 174-178.

\section{Assets from publication with us}

- Prompt Acknowledgement after receiving the article

- Thorough Double blinded peer review

- Rapid Publication

- Issue of Publication Certificate

- High visibility of your Published work

Website: www.actascientific.com/

Submit Article: www.actascientific.com/submission.php

Email us: editor@actascientific.com

Contact us: +919182824667 